\title{
Frequency Selective Surface Using the Metamaterial Property of the U-Shaped Strip
}

\author{
Mamoon A. Al-Atrakchii ${ }^{1}$, Khalil H. Sayidmarie ${ }^{2}$, Raed A. Abd-Alhameed ${ }^{3}$ \\ \{ maamoin91@gmail.com¹ ${ }^{1}$ kh.sayidmarie@gmail.com ${ }^{2}$, R.A.A.Abd@bradford.ac.uk ${ }^{3}$ \} \\ College of Electronic Engineering, Ninevah University, Mosul, Iraq ${ }^{1,2}$, \\ School of Engineering \& Informatics, University of Bradford, Bradford, UK ${ }^{3}$
}

\begin{abstract}
The frequency selective surfaces (FSS) are drawn great interests for their wide range of applications. In this paper, a simple and compact shape for the FSS is proposed, in which the resonant element is a conducting strip that is folded into the shape of the letter$U$ and placed on a dielectric sheet. The folding of the strip reduces the element length to $1 / 2$ of the effective wavelength, which is a considerable size reduction in comparison with other shapes. An example of the FSS was designed to reject the WiMAX frequency of 3.5 $\mathrm{GHz}$ and was investigated using the CST software package. The transfer characteristics were shown for the two cases of the vertical and horizontal polarizations of the incident electric field. The obtained rejection at the stopband was about $35 \mathrm{~dB}$. The obtained results are explained by considering the surface current distribution and electric field at the strip as well as the metamaterial behavior across the reject band. This approach gives better insight to understand the frequency selective property of the FSS.
\end{abstract}

Keywords: frequency selective surfaces (FSS), metamaterials, resonating element, bandstop.

\section{Introduction}

The Frequency Selective Surfaces (FSS) have been used to offer the properties of frequency-dependent reflection or transmission. An array of elements having a certain shape that is printed on a dielectric plate can highly reflect the incident e.m. waves across the certain frequency range. The FSS has been used in the RF and microwave regions of the electromagnetic (e.m.) wave spectrum and find many applications as in microwave ovens, shielding sensitive devices and circuits, antenna radomes and modern metamaterials [1]. The characteristics of the FSS, such as; resonance frequency, bandwidth, transfer characteristics, etc. are dependent on the shape of the unit-cell element and its dimensions in terms of the effective wavelength. The published works show that patches and/or slots in various shapes were used to achieve the metamaterial behavior or the FSS effects. Among the formerly used shapes were split ring [2], [3], square split-ring [4], V-Shape [5], spiral-S-shape [6], nested U-ring [7], and many other shapes [1], including the convoluted dipole [8].

The above shapes of slots or strips exhibit inductive and capacitive effects to the incident e.m. wave and resonate at certain frequencies. Therefore, their operation was explained in an analogous way to the microwave filters. The filtering properties of these structures were utilized to design various filters by embedding slots either in the microstrip line as in [9], or in the ground plane [5], or in both as in [10]. 
The FSS usually employ conducting strips placed on a dielectric sheet or slots in a conducting plane, whose sizes are a fraction of the wavelength of the incident wave. Thus, the metamaterial effect is inevitable, and the explanation of the frequency selective property can then be attributed to the resulting negative permittivity or permeability at a certain frequency range where the frequency selectivity appears [11], [12].

The U-Slot was used as a filter in antenna applications, as in [13], [14]. The presented design offers a very small width of the filter; thus, it was embedded into the microstrip feed line. Although the length of the proposed filter is about that of the square ring, the area of the presented filter is much smaller. In a recent publication [8], three shapes of the folded slot (Lshape, U-shape, and meandering line) were used for filtering applications. In a recent publication, an FSS structure of a square slot was investigated to relate the bandwidth of transmission with the phase range of the unit cell [15]. A bandpass FSS unit cell composed of two specially designed cavities with mixed electric and magnetic boundaries was presented in [16]. An array of shorting vias and complementary slots etched on both the top and bottom metallic patches constituted the boundaries. A bandstop or absorbing FSS having a unit cell in the form of a square ring circulating a rectangular atch that is truncated at two corners has been recently demonstrated [17]. The FSS covers a relatively wide range from $2.2 \mathrm{GHz}$ to $5.85 \mathrm{GHz}$ which includes the WLAN and WiFi bands, shows 17 to $21 \mathrm{~dB}$ shielding due to its polydimethylsiloxane (PDMS) substrate.

This paper investigates the frequency selectivity of a proposed U-shaped strip for FSS applications. It is shown that an array of U-shaped strips exhibits metamaterial properties when the effective wavelength is comparable to the size of the strips leading to a bandstop FSS structure. Better insight to explain the FSS performance is presented by examining the surface current distribution and electric field at the strip as well as the metamaterial behavior across the reject band. The rest of the paper is organized as follows. Section 2 presents the proposed FSS unit cell and simulation. Sections 3 and 4 present the analysis of the performance of the FSS for the two directions of the linear polarization, while section 5 lists the obtained conclusions.

\section{The FSS of the U-shaped strips}

The FSS proposed here is an array of U-shaped strips printed on a dielectric substrate. The FSS is studied using the unit cell approach (see Figure 1). The strip is folded into the U-shape to reduce its overall size while keeping the electrical length constant. Since at resonance, the electrical length is equal to the effective wavelength, then due to the folding, the length of the U-shaped strip will be about $1 / 2$ of the effective wavelength. This is a considerable size reduction in comparison with other shapes. The used unit cell is a square of $34 \mathrm{~mm}$ side length, which is 0.397 of the wavelength in air at the operating frequency of $3.5 \mathrm{GHz}$. The U-shaped copper strip is placed on an FR4 substrate of $0.8 \mathrm{~mm}$ thickness, dielectric of constant 4.4, and loss tangent of 0.025 . The copper plane thickness is $0.035 \mathrm{~mm}$, and the U-shaped strip has a width of $c=1 \mathrm{~mm}$, the total length of $71 \mathrm{~mm}$, where $\ell=30.5 \mathrm{~mm}$ and center part length $d$ is 10 $\mathrm{mm}$.

A linearly polarized uniform plane wave is assumed to be normally incident on the dielectric sheet with the copper U-shaped strips. The interaction of the incident wave with the copper strips can be studied into two cases. The first is when the electric field is parallel to the legs of the U-shaped strips. The other case is that when the Electric field (E-field) is perpendicular to the legs of the U-shaped strips. The CST Microwave Studio was used to simulate and study the performance of the designed FSS. The two cases of the linear polarization are investigated in the following two sections. 


\section{The electric field is parallel to the $\mathrm{U}$-strip ( $\mathrm{E} \| \mathrm{U}$ )}

As shown in Figure 1, the incident wave is assumed propagating along the Z-axis with its electric-field oriented along the Y-axis, i.e., parallel to the legs of the U-strip. The unit cell approach is used to represent the case of a 2-dimensional array of U-shaped strips. The proper boundary conditions for this case are Perfect Electric Conductor (PEC) perpendicular to the Yaxis and Perfect Magnetic Conductor (PMC) perpendicular to the X-axis. The input and output ports are placed along the Z-axis.

The characteristics (including reflection coefficient $S_{11}$, and transmission coefficient $S_{21}$ ) of the U-shaped strip FSS structure, such as the resonance frequency and stopband depend on the structural parameters of the U-shape strip. The strip length, strip width, and distance between the two legs of the strips are the parameters that control the transfer characteristics. In Figure 2, the simulated transfer characteristics for the U-shaped FSS filter are plotted for various slot lengths while keeping $d=10 \mathrm{~mm}, c=1 \mathrm{~mm}$ constants. The figure shows that as the strip length is increased, the resonance frequency decreases. The percentage change in the resonance frequency is inversely proportional to the change in length.

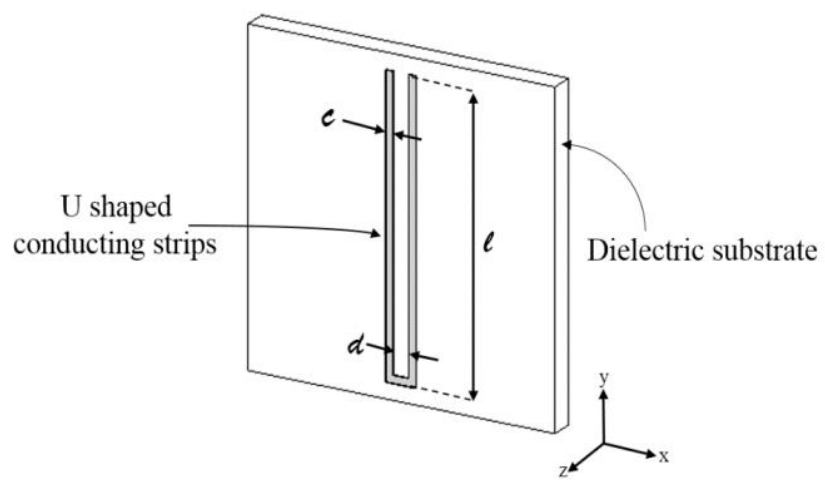

Fig. 1. The geometry of the unit cell of the proposed FSS structure using U-shaped strips.

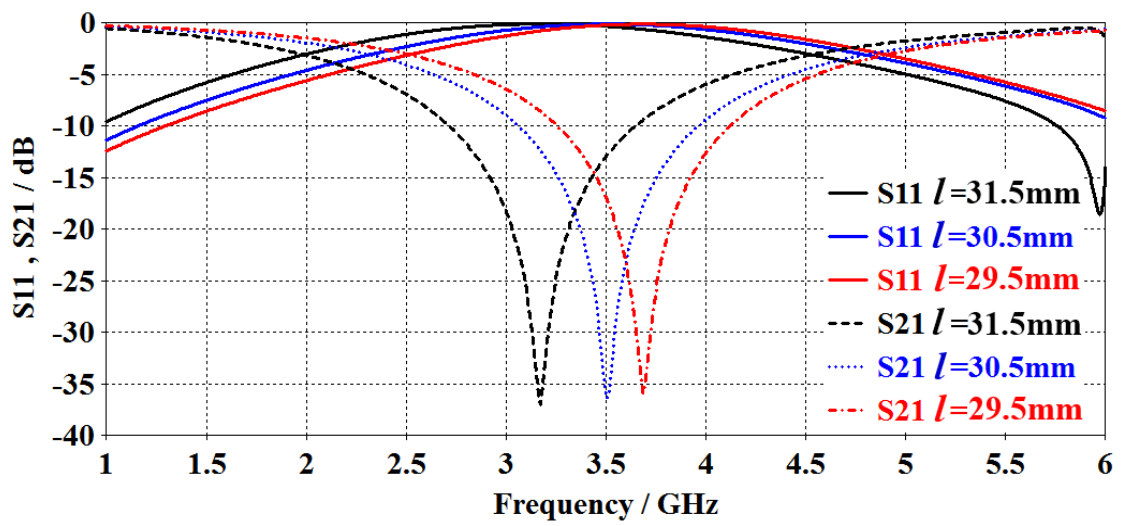

Fig. 2. Reflection coefficient $S_{11}$, and transmission coefficient $S_{21}$ of the U-shaped FSS structure for various values of the total length $l(d=10 \mathrm{~mm}, c=1 \mathrm{~mm})$. 
The transfer characteristics for strips of various widths were also simulated, and the computed results are shown in Figure 3 , when $\ell=30.5 \mathrm{~mm}$, and $d=10 \mathrm{~mm}$. The line width of the $\mathrm{U}$-shaped strip influences the resonance frequency and the width of the rejected band. The figure shows that a larger strip width results in a wider stopband, and slightly higher center frequency. When the strip width was changed by four folds (from $0.5 \mathrm{~mm}$ to $2 \mathrm{~mm}$ ), the center frequency changed by only $11.4 \%$. The influence of the strip width on the resonance frequency can be attributed to the mutual coupling between the two legs of the strip, which increases as the separation between the two legs increases.

Figure 4 shows the effect when modifying the separation distance between the two legs of the U-shaped strip while keeping the total U-shaped length unchanged. It can be noted that the resonance frequency slightly decreases (by about $12.2 \%$ ) when the separation distance was reduced from $13 \mathrm{~mm}$ to $7 \mathrm{~mm}$. Such an effect can be attributed to the mutual coupling between the two legs of the strip, where a larger separation distance $d$ leads to a lower mutual coupling.

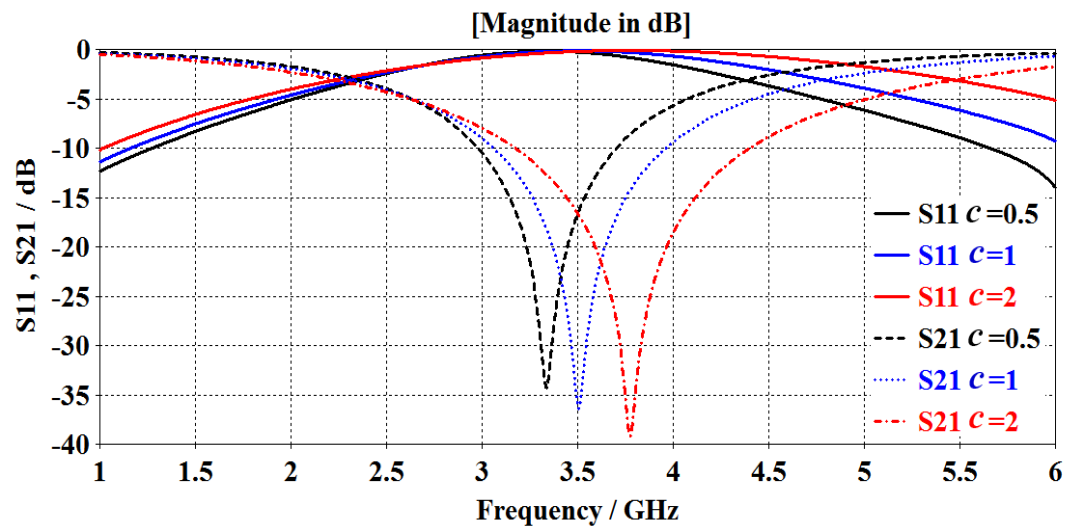

Fig. 3. Reflection coefficient $S_{11}$, and transmission coefficient $S_{21}$ of the U-shaped FSS structure for various line width $c(\ell=30.5 \mathrm{~mm}$ and $d=10 \mathrm{~mm})$.

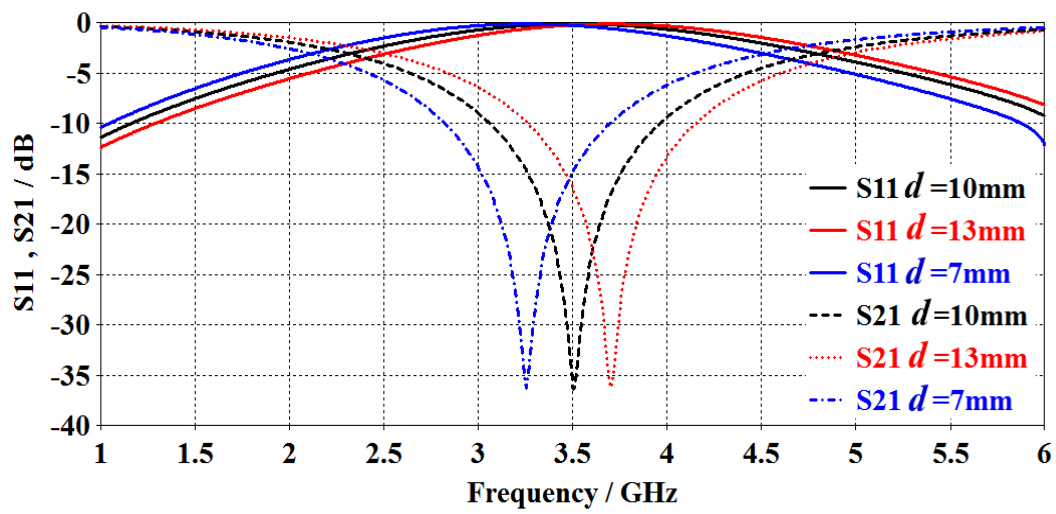

Fig. 4. Reflection coefficient $S_{11}$, and transmission coefficient $S_{21}$ of the U-shaped FSS structure for various center lengths $d(\ell=30.5 \mathrm{~mm}$ and $c=10 \mathrm{~mm})$. 
The effective permittivity and permeability of the FSS strip structure were calculated using the procedure described in [18], and the obtained results are shown in Figure 5. It can be seen that the effective permeability acquire negative values around the resonance frequency. The change in the effective permeability leads to a change in the impedance and hence affects the reflection coefficient. Thus, the shown metamaterial effect can explain the frequency sensitive behavior of the FSS structure.

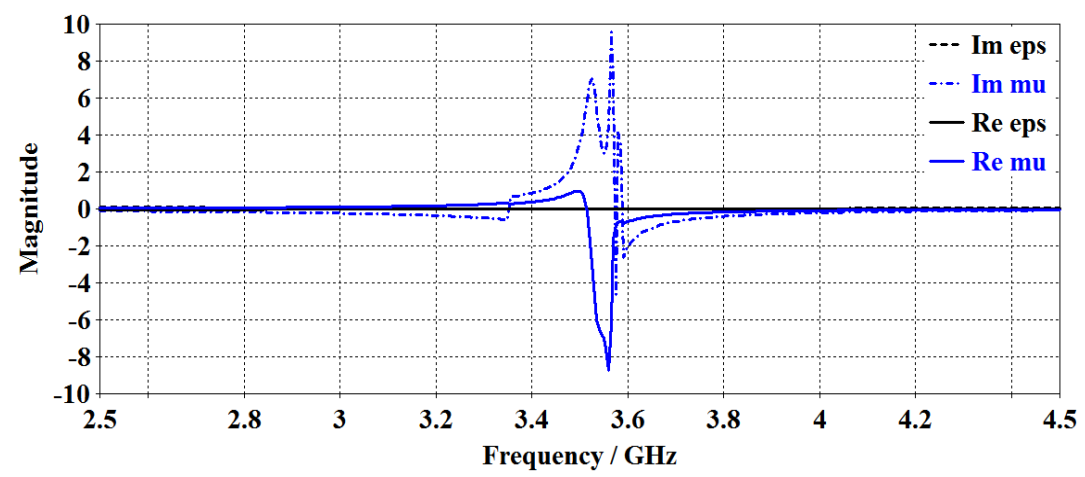

Fig. 5. Extracted effective permittivity and permeability of the U-shaped $(\ell=30.5 \mathrm{~mm}, c=1 \mathrm{~mm}$, and $d$ $=10 \mathrm{~mm})$. - real permittivity, ---- imaginary permittivity, - real permeability, ---- imaginary permeability.

For a better insight into the effect of the U-shaped strip on the propagation properties of the FSS structure for the above case, the electric field distribution along the U-shaped strip as well as the surface current density on the strip were studied. Figure 6 shows the electric field distribution around the U-shaped strip at the frequency of the reject band of $3.5 \mathrm{GHz}$ and another frequency in the passband, such as $5 \mathrm{GHz}$, while Figure 7 displays the surface current density for the corresponding two frequencies. For the frequency of $3.5 \mathrm{GHz}$, the electric field is concentrated along the two legs and the center of the U-shaped strip, while the current density has two peaks on the two legs. The distributions of the electric field and current density represent one guided-wavelength variation, as shown in Figures 6 and 7. At the $5 \mathrm{GHz}$ frequency, the electric field around the strip has an approximately uniform value of $8690 \mathrm{~V} / \mathrm{m}$, which is much lower than that $(21350 \mathrm{~V} / \mathrm{m})$ found for the $3.5 \mathrm{GHz}$ case. The current density has small values along the strip, as indicated by Figure 7-b. Thus, the U-shaped strip is not resonating at the 5 $\mathrm{GHz}$ frequency, and the incident wave penetrates the FSS structure with a low loss of about $3.9 \mathrm{~dB}$.

The average length $\mathrm{L}_{\mathrm{S}}$ of the U-shaped strip can be found from the geometry of Figure 1 as:

$$
\mathrm{L}_{\mathrm{S}}=2 \times(\ell-c / 2)+d+2 \times(c / 2)=2 \ell+d
$$

For the dimensions of the investigated U-shaped strip, the length of the strip, according to equation (1), and the wavelength at the frequency of $3.5 \mathrm{GHz}$ are the wavelength in air $\lambda_{0}=85.714 \mathrm{~mm}$, and the strip length $L_{s}=71 \mathrm{~mm}$. As Figures 6 and 7 show, the electric field and current density exhibit one wavelength variation along the strip, then one may conclude that $\mathrm{L}_{\mathrm{S}}=\lambda_{\mathrm{g}}=71 \mathrm{~mm}$. Since the effective wavelength $\lambda_{\mathrm{g}}$ and the wavelength in air $\lambda_{\mathrm{o}}$ are related by the effective permittivity $\varepsilon_{\text {re }}$ as: 


$$
\lambda_{\mathrm{g}}=\frac{\lambda_{0}}{\sqrt{\varepsilon_{r e}}}
$$

Then substituting into equation (2) for the above values of $\lambda_{\mathrm{g}}$ and $\lambda_{\mathrm{o}}$ yields $\varepsilon_{\mathrm{re}}=(85.714 / 71)^{2}$ $=1.46$

The above value is some way between the relative permittivity for air $\left(\varepsilon_{\mathrm{ro}}=1\right)$ and that for the FR4 substrate $\left(\varepsilon_{\mathrm{rs}}=4.4\right)$. It should be noted that the U-shaped strip is printed as a 2 dimensional array on the FR4 substrate, rather than there is a single strip as the case with printed antennas. For printed antennas, a rough estimate for the effective relative permittivity has been given by an average value of those for air and the substrate [14]:

$$
\varepsilon_{\mathrm{re}}=\left(1+\varepsilon_{\mathrm{rs}}\right) / 2
$$

which for the above substrate yields $\varepsilon_{\mathrm{re}}=2.7$.

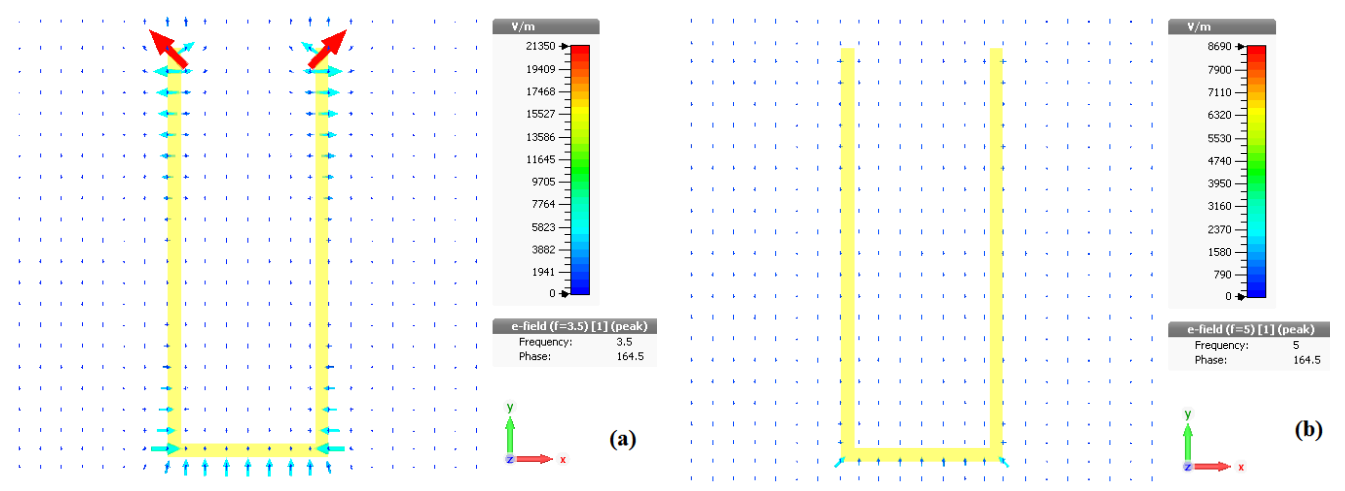

Fig. 6. Simulated E-field distribution around the U-shaped strip. (a) at $3.5 \mathrm{GHz}$. (b) At $5 \mathrm{GHz}$. The Efield of the incident wave is along the $\mathrm{Y}$-axis (E-field is parallel to the strip legs).
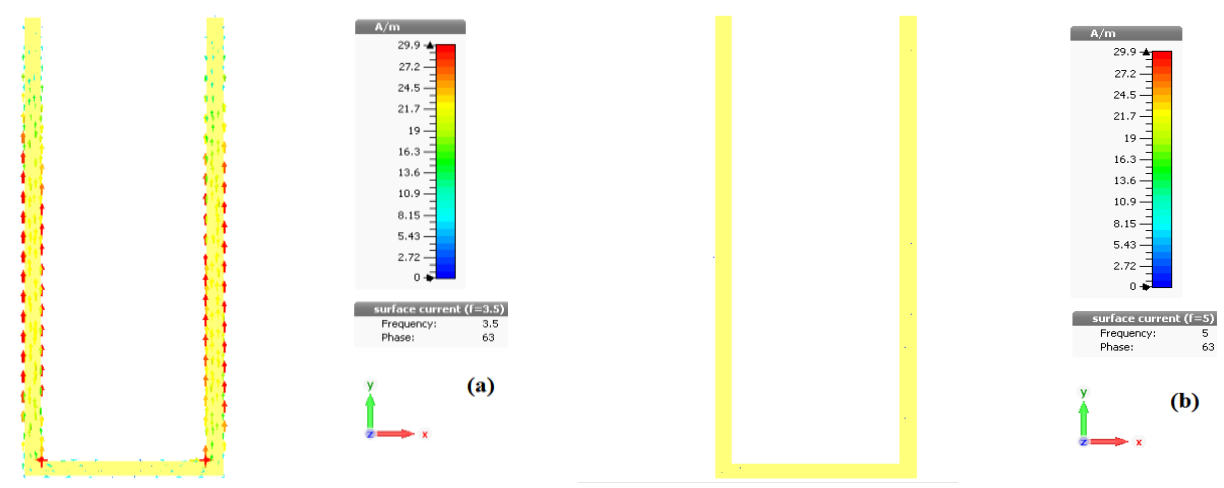

Fig. 7. Simulated current distribution on the U-shaped strip. (a) at $3.5 \mathrm{GHz}$. (b) At $5 \mathrm{GHz}$. The E-field of the incident wave is along the $\mathrm{Y}$-axis (E-field is parallel to the strip legs). 
A test was simulated by applying a plane wave in normal incidence to the FSS structure and calculating the radiation pattern on the other side. The directive gain of the U-shaped FSS was found to be $-9.4 \mathrm{~dB}$ at the stop frequency of $3.5 \mathrm{GHz}$, while it is $5.9 \mathrm{~dB}$ at the passband frequency of $5 \mathrm{GHz}$. This is a $15.3 \mathrm{~dB}$ discrimination in the transmitted signal level between the case of passband and rejectband.

\section{The electric field is perpendicular to the $U$-strip $(E \perp U)$}

In this case, the incident wave on the FSS screen is assumed linearly polarized, propagating along the $\mathrm{Z}$-axis, and has an E-field vector along the $\mathrm{X}$-axis, i.e., perpendicular to the strip legs. To simulate this case, the boundary conditions of the unit cell should be; PEC surfaces perpendicular to the $\mathrm{X}$-axis and PMC surfaces perpendicular to the $\mathrm{Y}$-axis. The obtained results from the simulation using the same dimensions of the strip that was studied in the former section $(\ell=30.5 \mathrm{~mm}, c=1 \mathrm{~mm}$, and $d$ is $10 \mathrm{~mm})$ are shown in Figure 8 . The result also indicates a bandreject property as with the former case, but at $1.65 \mathrm{GHz}$ frequency, with a reflection coefficient less than $-1 \mathrm{~dB}$, and the transmission coefficient is $-18 \mathrm{~dB}$. The reject band feature is repeated at a higher frequency of $5.2 \mathrm{GHz}$, which is about 3.15 of the first frequency of $1.65 \mathrm{GHz}$. In this case, the E-field is perpendicular to the log parts (legs) of the U-shaped strip, and it is parallel to the short part (central part) of the strip. This is an opposite orientation to that studied in the former section; thus, it is expected to yield different behavior.

By keeping the same boundary conditions, and decreasing the total length of the strip to 32 $\mathrm{mm}$ (where $\ell=13 \mathrm{~mm}, d=6 \mathrm{~mm}$, and $c=1 \mathrm{~mm}$ ), the resonance frequency is now shifted to $3.5 \mathrm{GHz}$. The performance of the FSS structure is now enhanced (higher reflection coefficient $\mathrm{S}_{11}=-0.5 \mathrm{~dB}$ and lower transmission coefficient $\mathrm{S}_{21}=-24 \mathrm{~dB}$ ) at the center frequency, as shown in Figure 9.

The effective permittivity and permeability of the FSS strip structure were calculated using the procedure described in [18], and the obtained results are shown in Figure 10. It can be seen that the effective permittivity acquires negative values around the resonance frequency. The change in the permittivity leads to a change in the impedance and hence affects the reflection coefficient. This is a metamaterial property that is responsible for the frequency selective response of the FSS U-shaped structure.

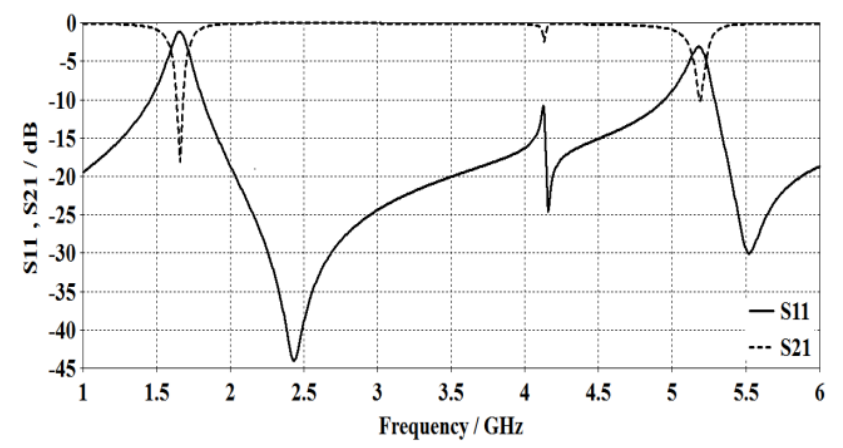

Fig. 8. Reflection, and transmission coefficients $S_{11}, S_{21}$ of U-shaped strip when the E-field is $X$ directed $(\ell=30.5 \mathrm{~mm}, c=1 \mathrm{~mm}$, and $d=10 \mathrm{~mm})$. 


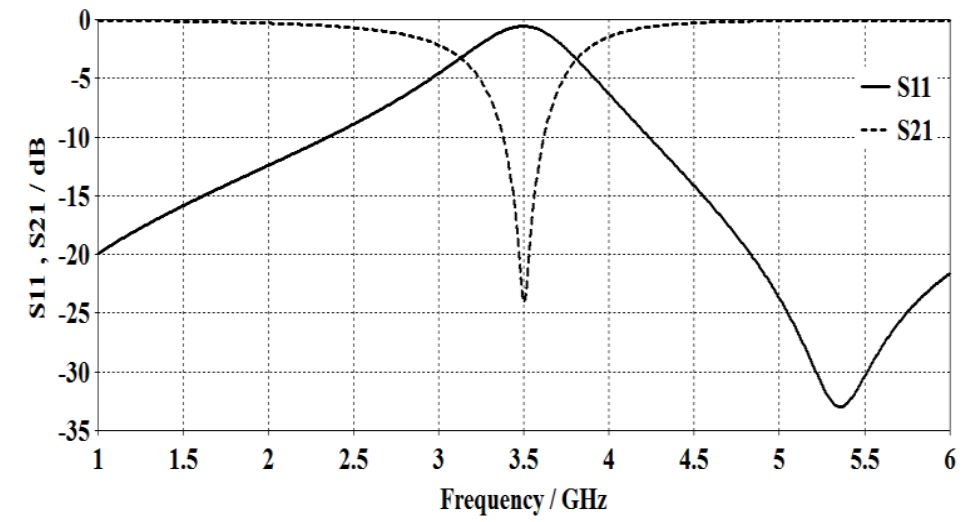

Fig. 9. Reflection, and transmission coefficients $S_{11}, S_{21}$ of the U-shaped strip when the E-field is $X$ directed $(\ell=13 \mathrm{~mm}, c=1 \mathrm{~mm}$, and $d=6 \mathrm{~mm})$.

The electric field distribution and the surface current density around the U-shaped strip were studied, and the obtained results are shown in Figures 11 and 12. Figure 11 shows the electric field distribution around the U-shaped strip at the frequencies of the reject band $3.5 \mathrm{GHz}$ and the passband $5 \mathrm{GHz}$. For the $3.5 \mathrm{GHz}$ frequency, the E-field near the two legs of the Ushaped strip are in maximum and tends to zero at the center of the legs. Such change represents a half wavelength variation, and thus the strip is resonating. At the same time, Figure 12 shows that the surface current density achieves maximum value at the center of the strip, and confirms the half-wavelength variation along the strip. At the $5 \mathrm{GHz}$ frequency E-field intensity near the strip and the surface, current density has much lower values compared to those for the $3.5 \mathrm{GHz}$ case. Thus, the $\mathrm{U}$ shaped strip shows no resonance at the passband.

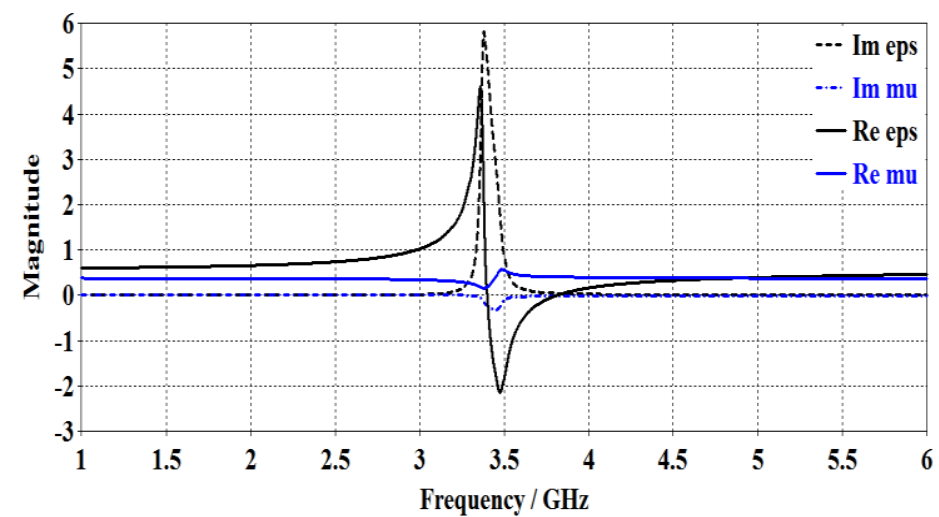

Fig. 10. Extracted effective electric permittivity and magnetic permeability of U-shaped $(c=1 \mathrm{~mm}, \ell=13$ $\mathrm{mm}$, and $d=6 \mathrm{~mm}$ ). - real permittivity, ---- imaginary permittivity, — real permeability, ---- imaginary permeability.

As Figures 11 and 12 show the results for the case of $(c=1 \mathrm{~mm}, \ell=13 \mathrm{~mm}$, and $d$ is $6 \mathrm{~mm})$, i.e. the total length of the strip is $32 \mathrm{~mm}$. Recalling the analysis of the former section where $\mathrm{L}_{\mathrm{S}}=0.5 \lambda_{\mathrm{g}}=32 \mathrm{~mm}$, Thus, $\lambda_{\mathrm{g}}=64 \mathrm{~mm}$, and using equation (2) the effective relative permittivity 
will be $\varepsilon_{\mathrm{re}}=(85.714 / 64)^{2}=1.79$. The difference from that obtained for the first case when the Efield is parallel to the legs of the U-shaped strip can be attributed to the fact that the relations between the conducting strip and the orientation of the electric field are different for the two cases. The permittivity is a relation between the electric displacement and the electric field intensity. Therefore, the estimated values of the effective permittivity are influenced by the spatial relation between the electric field vector and the conducting strip.

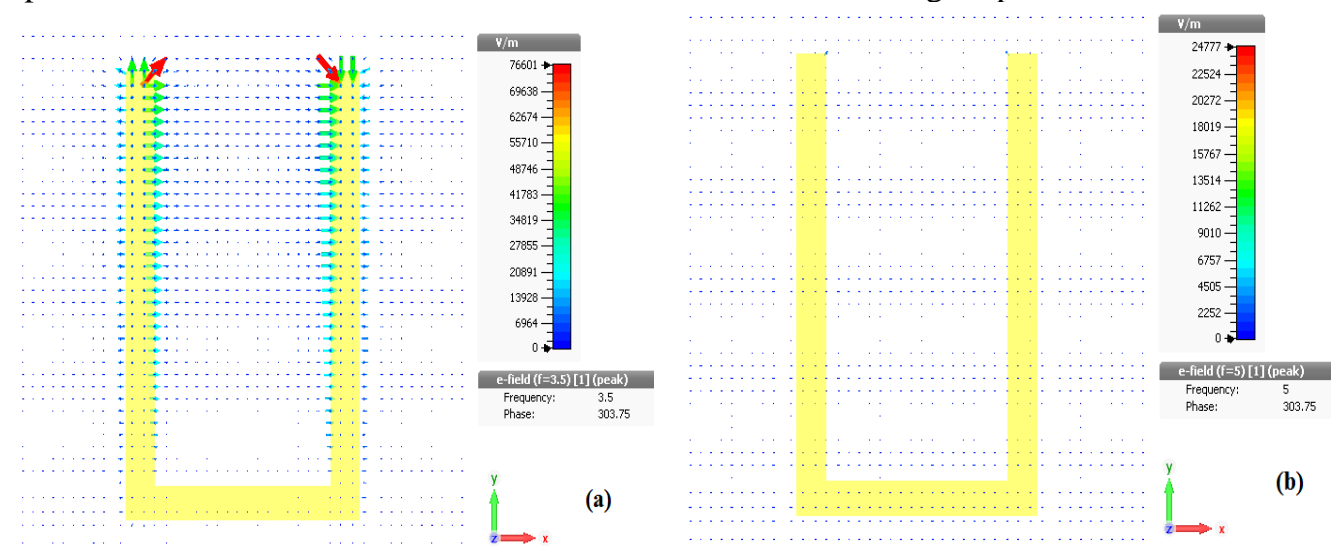

Fig. 11. Simulated E-field distribution around the U-shaped strip. (a) at $3.5 \mathrm{GHz}$. (b) At $5 \mathrm{GHz}$. The Efield of the incident wave is along the $\mathrm{X}$-axis (E-field is perpendicular to the strip legs).
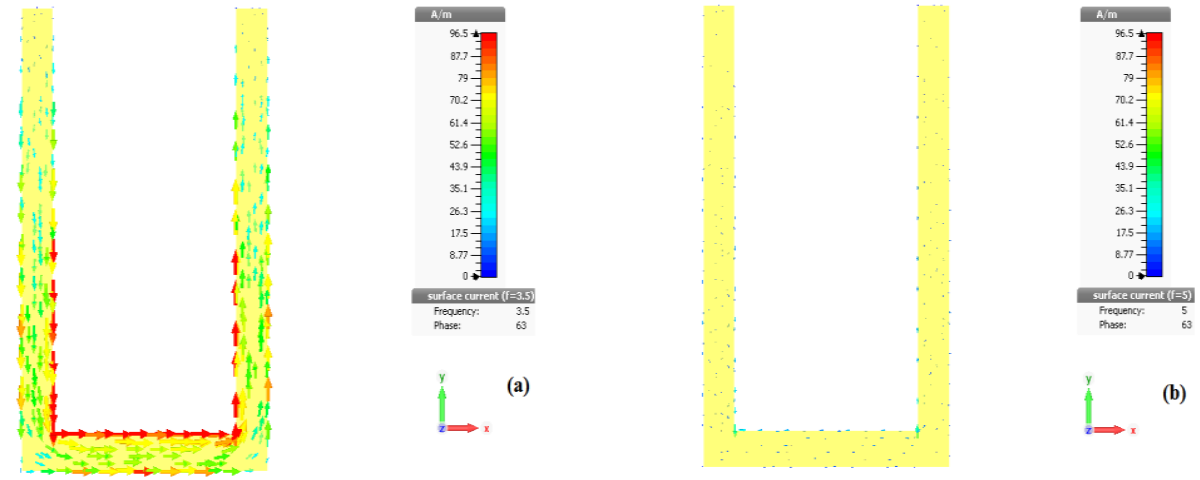

Fig. 12. Simulated surface-current distribution on the U-shaped strip. (a) at $3.5 \mathrm{GHz}$. (b) At $5 \mathrm{GHz}$. The E-field of the incident wave is along the $\mathrm{X}$-axis (E-field is perpendicular to the strip legs).

\section{Comparison with other FSS works}

There are various designs of the FSS which differ vastly in their characteristics and sizes in terms of the wavelength at the operating frequency, depending on the required application. Due to the folding of the strip into U-shape, the length of the folded slot becomes slightly less than $1 / 2$ the effective wavelength at the center of the stopband. Since the effective wavelength is 
always smaller than that in air, then the size of the strip is always smaller than $1 / 2$ the wavelength in air. The characteristics of the proposed filter are compared in Table 1 with those of other FSS designs operating at nearby bands that are published in the literature. It can be seen that the performance of the proposed filter is comparable to those other designs, while it has simple design and low-cost substrate.

Table 1 Comparison of the characteristics of the proposed FSS with other published designs.

\begin{tabular}{|c|c|c|c|c|c|c|c|}
\hline Ref. & $\begin{array}{l}\text { Applicatio } \\
\mathrm{n}\end{array}$ & $\begin{array}{l}\text { Freq. } \\
\text { (GHz) }\end{array}$ & $\varepsilon_{\mathrm{r} / \tan \delta}$ & $\begin{array}{l}\text { Cell } \\
\text { dimensions } \\
\lambda_{\mathrm{o}} \times \lambda_{\mathrm{o}} \\
\end{array}$ & $\begin{array}{l}\mathrm{S}_{11} \\
(\mathrm{~dB})\end{array}$ & $\begin{array}{l}S_{21} \\
(d B)\end{array}$ & shape \\
\hline [8] & Band-pass & 1.64 & $\begin{array}{l}\mathrm{F} 4 \mathrm{~B} \\
2.65\end{array}$ & $0.03 \times 0.03$ & N.A. & $\sim 0$ & Convoluted dipole \\
\hline [11] & Absorber & $5.5-18$ & 1.5 & $0.57 \times 0.57$ & -10 & $\begin{array}{l}\text { absorb } \\
\text { er }\end{array}$ & $\begin{array}{l}\text { Crisscross \& fractal } \\
\text { square patch }\end{array}$ \\
\hline [15] & Band-pass & 3.86 & $4.4 / 0.02$ & $0.22 \times 0.22$ & & $\sim 0$ & Square-ring slot \\
\hline [16] & Band-pass & 4.58 & $3.7 / 0.0029$ & $0.32 \times 0.32$ & & 0.37 & $\begin{array}{l}\text { Two cavities with } \\
\text { mixed electric and } \\
\text { magnetic boundaries }\end{array}$ \\
\hline [17] & $\begin{array}{l}\text { Band- } \\
\text { Stop }\end{array}$ & $\begin{array}{l}2.2- \\
5.85\end{array}$ & $\begin{array}{l}\text { sandwich of } \\
\text { ITO-PET- } \\
\text { PDMS- } \\
\text { PET-ITO } \\
\end{array}$ & $\begin{array}{l}0.53 \times 0.53 \mathrm{x} \\
0.15\end{array}$ & & $\begin{array}{l}-17 \text { to }- \\
21 \mathrm{~dB}\end{array}$ & $\begin{array}{l}\text { Two L-shaped strips + } \\
\text { truncated square }\end{array}$ \\
\hline $\begin{array}{l}\text { This } \\
\text { work }\end{array}$ & $\begin{array}{l}\text { Band- } \\
\text { reject }\end{array}$ & 3.5 & $4.4 / 0.025$ & $0.46 \times 0.46$ & $\sim 0$ & -30 & U-Shaped strip \\
\hline
\end{tabular}

\section{Conclusions}

It has been demonstrated that a conducting strip having U-shape can be utilized in the design of FSS for band rejection. The folding of the strip into the U-shape offers size reduction, as its length is smaller than $1 / 2$ the effective wavelength. The analysis and simulations showed that the band-reject frequency is related to the resonating U-shaped strip, where the length of the strip is half or one effective wavelength. This depends on the orientation of the electric field of the incident wave with respect to the $\mathrm{U}$ strip. The obtained rejection at the stopband was about $35 \mathrm{~dB}$. Examination of the surface current distribution and electric field at the strip, as well as the metamaterial behavior across the reject band gives better insight into the performance of the FSS. The FSS of the U-shaped strips showed metamaterial behavior at frequencies around the stopband. 


\section{References}

[1] Anwar, R. S., Mao, L. and Ning, H.: Frequency selective surfaces: A Review. Applied Science. 2018, 8, 1689; doi:10.3390/app8091689.

[2] Pan, P. Wu, Q. Meng, F. Y. Zhang, F., and Lee, J.: A novel compact left-handed transmission line with improved split-ring resonators. APMC Asia-Pacific Microwave Conference Proceedings, pp. 14, (2007).

[3] Gil, M., Bonache, J. Selga, J. Garcia-Garcia, J., and Martin, F.: High-pass filters implemented by composite right/left-handed (CRLH) transmission lines based on complementary split rings resonators (CSRRs). PIERS Online. Vol. 3, No. 3, pp. 251-253 (2007).

[4] Fallahzadeh, S. and Tayarani, M.: A compact microstrip bandstop filter. Progress in Electromagnetics Research Letters. Vol. 11, pp. 167-172 (2009).

[5] Ekmekçi, E., and Turhan-Sayan, G.: Investigation of effective permittivity and permeability for a novel V-shaped metamaterial using simulated S-parameters. 5th International Conference on Electrical and Electronics Engineering. pp. 251-254 (2007).

[6] Amiri, N., Forooraghi, K. and Atlasbaf, Z.: Design and simulation of a novel double negative metamaterial. Asia Pacific Microwave Conference. pp. 728-730 (7-10 Dec. 2009).

[7] Turkmen, O., Ekmekci, E. and Turhan-Sayan, G.: Nested U-ring resonators: a novel multi-band metamaterial design in microwave region. IET Microwaves, Antennas \& Propagation. Vol. 6, No. 10, pp. 1102-1108 (2012).

[8] Wang, M., Zhao, L., Wang, J., and et al.: A low-profile miniaturized frequency selective surface with insensitive polarization. ACES Journal, Vol. 33, No. 9, (Sept. 2018).

[9] Li, Y., Luo, S. and Yu, W.: A compact tunable triple stopband filter based on different defected microstrip structures. ACES Journal, Vol. 33, No. 7, pp. 752-757 (July 2018).

[10] Belkadi, B., Mahdjoub, Z. and Seddiki, M. L.: Design and analysis of dual-band rejection microwave filter employing SRR. 5th ICEE-B, Int. Conf. Electr. Eng. -Boumerdes. (2017).

[11] Sun, L., Cheng, H. Zhou, Y. and Wang, J.: Broadband metamaterial absorber based on coupling resistive frequency selective surface. Optic Express. Vol. 20, no. 4, pp. 4675-80 (Feb. 2012).

[12] Song, K., and Mazumdar, P.: Design of highly selective metamaterials for sensing platforms. IEEE Sensors Journal. Vol. 13, No. 9, pp. 3377 - 3385 (2013)

[13] Sayidmarie, K. H., and Najm, T. A.: Performance evaluation of band-notch techniques for printed dual-band monopole antennas. International Journal of Electromagnetics and Applications. Vol. 3, No. 4, pp. 70-80 (2013).

[14] Yahya, L. S., Sayidmarie, K. H. Elmegri, F. Abd-Alhameed, R. A.: Crescent-shaped doublemonopole antennas with reduced coupling for WLAN and WIMAX applications. IEEE Conference on Internet Technologies and Applications (ITA). pp. 393 - 398, Wrexham, UK (8-11 Sept. 2015).

[15] Singh C., Jha K.R., Sharma S.K., Jibran Z.A.P., Singh G.: Design of a wideband square slot bandpass frequency-selective surface using phase range analysis. Engineering Reports. 2020;e12085.

[16] Yang, L., Wei, X., Yi, D., and Jin, J.: A bandpass frequency selective surface with a low crosspolarization based on cavities with a hybrid boundary. IEEE Transactions on Antennas and Propagation, Vol. 65, No. 2, pp. 654-661, (Feb. 2017).

[17] Wu, ., Wang, J., Lai, S., Zhu, X. and Gu, W., : A transparent and flexible microwave absorber covering the whole WiFi waveband. AIP Advances 9, 025309 (2019); doi: 10.1063/1.5083102.

[18] Szabo, Z., Park, G. Hedge, R., and Li," E.: A unique extraction of metamaterial parameters based on Kramers-Kronig relationship. IEEE Transactions on Microwave Theory and Techniques. Vol. 58, No. 10, pp. 2646-2653 (2010). 